\title{
Efficient Utilization of Cooperative Spectrum Sensing (CSS) in Cognitive Radio Network (CRN) Using Non-Orthogonal Multiple Access (NOMA)
}

BALACHANDER T ( $\square$ balachat2@srmist.edu.in )

SRM Institute of Science and Technology https://orcid.org/0000-0002-9937-6942

Mukesh Krishnan M B

SRMIST: SRM Institute of Science and Technology

\section{Research Article}

Keywords: CRN, NOMA, 5G Wireless communication, spectrum sensing, spectrum sharing

Posted Date: March 2nd, 2021

DOl: https://doi.org/10.21203/rs.3.rs-238720/v1

License: (c) (1) This work is licensed under a Creative Commons Attribution 4.0 International License.

Read Full License

Version of Record: A version of this preprint was published at Wireless Personal Communications on August 11th, 2021. See the published version at https://doi.org/10.1007/s11277-021-08776-7. 


\title{
EFFICIENT UTILIZATION OF COOPERATIVE SPECTRUM SENSING (CSS) IN COGNITIVE RADIO NETWORK (CRN) USING NON-ORTHOGONAL MULTIPLE ACCESS (NOMA)
}

\author{
T.Balachander ${ }^{1}$, Dr. M.B.Mukesh Krishnan ${ }^{2}$ \\ 1Department of Computer Science and Engineering, School of Computing, \\ College of Engineering and Technology, SRM Institute of Science and Technology, \\ Kattankulathur, Chennai, India \\ 2Associate Professor Department of Information Technology, School of Computing, \\ College of Engineering and Technology, SRM Institute of Science and Technology, \\ Kattankulathur, Chennai, India \\ ${ }^{1}$ balachat2@srmist.edu.in, ${ }^{2}$ mukeshkm@srmist.edu.in
}

\begin{abstract}
In the recent past, efficient cooperative spectrum sensing and usage are playing a vital role in wireless communication because of the significant progress of mobile devices. There is a recent surge and interest on Non-Orthogonal Multiple Access (NOMA) focused on communication powered by wireless mode. In modern research, more attention has been focused on efficient and accurate Non-Orthogonal Multiple Access (NOMA). NOMA wireless communication is highly adapted with Cognitive Radio Network (CRN) for improving performance. In the existing cognitive radio network, the secondary users could be able to access the idle available spectrum while primary users are engaged. In the traditional CRN, the primary user's frequency bands are sensed as free, the secondary users could be utilized those bands of frequency resources. In this research, the novel methodology is proposed for cooperative spectrum sensing in $\mathrm{CRN}$ for $5 \mathrm{G}$ wireless communication using NOMA. The higher cooperative spectrum efficiency can be detected in the presence of channel noise. Cooperative spectrum sensing is used to improve the efficient utilization of spectrum. The spectrum bands with license authority primary user are shared by Secondary Users (SU) by simultaneously transmitting information with Primary Users (PU). The cooperative spectrum sensing provides well under the circumstances that the different channel interference to the primary user can be guaranteed to be negligible than an assured thresholding value. The Noisy Channel State Information (CSI) like AWGN and Rayleigh fading channels are considered as wireless transmission mediums for transmitting a signal using Multiple-Input-Multiple-Output (MIMO) NOMA to increase the number of users. The proposed NOMA is fascinated with significant benefits in CRN is an essential wireless communication method for upcoming 5G technology. From experimental results it has been proved that the novel methodology performance is efficient and accurate than existing methodologies by showing graphical representations and tabulated parameters.
\end{abstract}

Keywords: CRN, NOMA, 5G Wireless communication, spectrum sensing, spectrum sharing 


\section{Introduction}

An energy efficient transmission of CRN is considered while every secondary transmitter (ST) extracts radio frequency (RF) signals through primary transmitters (PT) in the neighbourhood which conveys information to its secondary receiver (SR), which encounters co-channel interference through PT. A constructive association is found amidst the ST energy collected and the SR cumulative intrusion, which indicates that if there is a heavy intervention in the SR, ST will have a higher chance of collecting sufficient energy. A transmission technique based on interruption threshold for STs is introduced to utilize the significant connection, to secure ancillary transmissions as well as maximize the source of power extracted. The energy state of each ST is represented as a discrete form Markov chain (DTMC) fixed state and derives the interpretation of ancillary spatial bandwidth as a function of likelihood of transmission and propagation likelihood. The influence of the interference threshold and the accumulation of PTs on the second strategy throughput was investigated to maximize the quantitative throughput in addition to provide advice on the best specification of these 2 factors [1].

Probabilistic Cognitive radio is intended to provide a cognitive software defined system characterized by linear programming Perceptual Maps. To solve many good problems of this learning model, first, basic architecture adaptations of Complicated Cognitive Maps are introduced. Second, the appropriate solvent of a networking architecture specified by the software is addressed and evaluated. Finally, to test the feasibility and transmission capacity of the advanced CRN and the capability to make a function respond regardless of any artificial and external interference, simulation of a model heterogeneous network is given. The integration of software-defined infrastructure and application development of network functions is needed for this method. Additionally, without human involvement, such a new dynamic network system would pose more questions and issues to be overcome both aggressively and constructively. To do this, the approach to the application and execution of artificial intelligence has been found by industry and academia. Therefore, most definitely, cognitive incredible would be 5G (and specifically beyond $5 \mathrm{G}$ ) systems. The channel maximization from primary transmitter to the receiver node is a vital interface factor that holds importance for the coherent functioning of CRN. Two new measurement methods for the primary channel gain are developed by using the received signals directly based on the method of spectrum sensing. Cognitive radio calculation is done seperately, without the participation of the primary user. The fading networks of Rayleigh and Nakagami-m are also taken into consideration. The estimators introduced are much easier and less stringent than the estimation methods that are in place [3].

In an appropriate CRN, the mutual NOMA method of incomplete sequential interference cancellation (SIC) is investigated. Taking into account that the channel parameters obey the Gaussian distributions among primary source and secondary collecting points, an approximate closed-form is extracted from the exact likelihood of interruption for each secondary recipient. Also, 1) It is derived from cumulative calculations for the noisy fading channels when the interference threshold rises to the peak and 2) when the transmission variables at the secondary source and transfer go over to the peak [4]. An integrated development to stable and time-efficient sensor networks is offered by routing protocol Cognitive capacity, however, takes resource cognitive radio sensor networks (CRSNs) to a very challenging problem as high energy usage is needed for CSS and false access of signal transmission. Due to its effect on resource wireless communication protocol, the significant layer medium access control (MAC) is important for CRN. A brief declaration of the semantic MAC (S-MAC) specification for CRSNs is proposed in this work. 
The clever mix of quick constitution sampling and duplicitous forwarding is S-MAC's improvement. As a result, stable and easy spectrum connectivity may be enabled by SPC-MAC while reducing energy usage.

Besides, without having any traditional control networks, SPC-MAC is a circulated cognitive radiospecific S-MAC protocol. For SPC-MAC, the procedure modeling is done robustly [5]. Centered on the idea for wireless downlink non-orthogonal multiple access (NOMA) systems, a new value power allocation approach is applied. Secondly, the constrained methodology is provided in the case of two users and it is shown that the transmission power can be allocated to all subscribers and only to those with a stronger channel condition. Then, depending on the methodology developed for the two users, an exponential approach is applied for more than two users' instances. An innovative NOMA power allocation methodology based on price is introduced. For the case of two users, the optimum solution and efficient power allocation method are explained to multi-users with the confined approach. For more than two user instances, an incremental power allocation method is then proposed which is based on the method for two users [6]. An angular velocity movement multiplexing system s introduced with NOMA to increase the user and sum channel properties of the downlink NOMA for many devices. The implementation and sum characteristics of the NOMA-MIMO conceptual process are subjected to analysis and distinguished with new following current systems such as the NOMA MIMO system and the orthogonal multiplexed MIMO model implementation.

To increase the channel capability of downlink NOMA wireless contact, NOMA-OAM-MIMO was suggested. The outcome study revealed that by combining higher numbers of OAM modes, the proposed NOMA-OAM-MIMO scheme with numbers of Tx, Rx UCAs, and OAM mode combination (Case 1) has surprisingly greater system throughput than traditional schemes [7]. A new hierarchical option of multiple access technologies is proposed between orthogonal multiple access (OMA) and non-orthogonal multiple access (NOMA) methods. A combined resource allocation issue is developed for this configuration where a new group of access technology based on selection criteria are assigned for each user, along with power and subcarrier, depending on the channel state of each user. To to be conscious of the rate and expense of access technology, a novel functionality is defined. This expense represents both the complexity of repeated termination of interruption and the difficulty sustained to ensure the optimal amount of bit error. This nonlinear element would necessarily define the differentiation between OMA and NOMA. A corresponding convex replication is developed in which, due to the non-convexity of the suggested resource allocation solution, an iterative algorithm is applied in two steps. During the first process, the problem is translated into a matrix vector integer linear programming, named the array of communication systems, and during the second process, a non-convex problem, related to power allocation, is addressed by computing the variance of differential equations (DE). Furthermore, the closed-form approach for power allocation is achieved in the subsequent process. Simulation findings shows that suggested latest distributed access technology deployment exceeds single-technology NOMA multiple access solutions for varied network efficiency metrics such as cost. Non-orthogonal multiple access (NOMA) for 5G networks is appraised as the most optimal multiple access (MA) approaches that can offer low bandwidth, fast device throughput, and massive convergence [8]. A cognitive radio (CR)-NOMA channel estimation network is identified, where ancillary data interacts with M NOMA supplementary destination users (SDUs) under uncertain channel state information circumstances by implies of a definitively diagnose secondary relay.

For NOMA, in a linear model, standard Outage Likelihood (OL) parameters are provided. Standardized end-to-end OP operations in confined categories are created for the DF-based surface CRN 
OMA channel allocation systems with M SUs. The theoretical outcomes were verified by simulations obtained via Monte Carlo. Next, the influence from inaccurate CSI on the performance of the unit was analyzed. It has been inferred from statistical results that a detailed evaluation of PA parameters will ensure the consistency of the performance of NOMA users. Moreover, OP quantitative findings for CR-NOMA and CR-OMA highlighted that the performance of NOMA system is optimal when compared wirh Orthogonal Multiple Access including and exculding the deployment of OMA [9]. Downlink erosion and efficiency reliability in the secondary structure of cognitive radio assisted NOMA networks. Both the MA method and NOMA method are evaluated with respect to the state of each user's configuration phase. In terms of performance, the sealed descriptions of the outage probability for two users are selected based on the transmitting signal-to-noise ratio (SNR) in the main source and the contact user interference threshold. To accomplish further information, an exponential analysis of the disrupted probability in the high SNR method is given. Optimum throughput offers a clear idea when calculating the power allocation function. Besides, power allocation variables, objective thresholds, and transmitting SNR is calculated to achieve high equal outage [10].

\section{The State of Art for Spectrum Sensing Models}

Papadopoulos Athanasios et. al [11] has explored transmitting strategies for a conceptual cooperative communication system, i.e. a CRN is a sensor node that will serve as a relay for data conveyed by the primary user, thus enhancing the efficient transmission of the primary user and gaining more possibilities of communication aimed at their particular communications. In particular, the ability to enhance the overall efficiency of the system is explored by the use of network coding strategies. The aim is to do this by only directly impacting primary user communications, notably: 1) eliminating cognitive radio activities at the primary converter, thus eliminating raising its storage capacity and retaining high specificity, 2) holding the packet sequence could be collected by the particular signal receiver the identical as in the noncooperative condition, then 3 ) bringing stochastically time of packet arrival. In terms of the accomplished throughput area, a cognitive radio algorithm is explored and secondary user bandwidth is seen to be expanded qualified to the situation where the subordinate source gatherings as an elementary receiver, separation the primary user transitory constancy unaffected. A remarkable characteristic of this methodology is that it functions despite channel and packet arrival rate statistics information.

Chaoying Yuan et. al [12] have studied a cooperative communicate collection system discretion ability using NOMA. A new cooperative NOMA framework was developed. Here tapping signals are deliberately transmitted by the source while the relay is transmitting, thereby improving the reliability of anticipated contact links. In the presence of unauthenticated, closed-form classifications for the hyperbolic geometry privacy rate are continued. In a more accurate signal-to-noise ratio (SNR) gaining system, that gives comprehension on steady NOMA communication, exponential estimated peers for the hyperbolic geometry primary mechanisms are formed. The important situation in which NOMA can outclass orthogonal multiple access (OMA) with respect to secrecy rate is exposed by simulation solution.

Muhammad Ahsen et. al [13] have proposed a wireless communication methodology strip-shaped multihop diverse ad hoc wireless sensor network was investigated which maeks use of a temporal Poisson point processing (PPP) and resourceful stochastic geometry. For distribution through the multi-hop system, where mutual communications are used at each hop, the decode-and-forward method is called. An empirical solution is given at an appropriate moment from a channel of points broadcast in the previous hop for the 
probability density function of the received power, used to define transmission efficiency of the given network. The power generated at a point happens to become a potentially similarly stochastic activity due to arbitrary path loss and an improper Rayleigh fading channel. The occurrences of one-hop output probability and propagation range is investigated for multiple distributed parameters. By analyzing PPP thinning, an energy efficiency approach is often applied and its efficiency is calculated with regard to the amount of energy saved.

Muhammad Farhan Sohail et. al [14] have suggested a NOMA energy system for the configuration of UAVBS control. The criteria for maximizing data symbols per unit power consumption of the UAV-BS is defined in the sense of a multi-quality of service (QoS) environment. Besides, the measurement of efficiency takes into account the configuration of energy needed for transmission and hovering phase of the UAV-BS signal. The established Non-Linear Fractional Problem (NLFP) as a function of wireless communication and acceleration is resolved by making use of a competitive advantage approach that is dependent on a nested framework. Investigation was done on the approach to user modeling provides an insight on its effect on the minimal theoretical elevation range of the UAV BS. As a consequence, a machine based perspective is structured to make the objective function dependent on the given subclass of user types. The quantitative analysis of the novel system indicates a significant and proportional increase in energy efficiency of the NOMA derived UAV-BSS based scheme relative to the baseline configuration of OMA. The improved energy efficiency thus helps the UAV-BS to have enhanced coverage.

Zhiyuan Yu et. al [15] has proposed two distinct multiple access (NOMA) method of mutual relaying. In the first phase, a base station (BS) combines the near-user U1 and far-user U2 signals sequentially and then communicates the superimposed pulse to $\mathrm{U} 1$ and multiple transmissions. U1 tries to decode the required information by treating the signal from $\mathrm{U} 2$ as an interference. By analyzing and suspending the U1 signal in a shared zone between AP and U2, several relays proceed to decode the U2 signal. In the second phase, an accurate relay is used to propel the far-user signal. Meanwhile, if U1 in the first phase attempts to decrypt its desired signal, it can try to decode and interrupt the far-user signal to better decode its desired signal accordingly. The system analyzes efficacy and discusses the effects of multiple variables.

Guo Cheng et. al [16] have suggested the interference cancellation methodology called Successive Interference Cancellation (SIC) in the NOMA system as the de decoding process. However, for the multiple access example, SIC also allows the matched users to have substantially diverse found energy levels. The parity and outage likelihoods are difficult due to the matched primary users are similar to each other of secondary users. A new encryption strategy based on correlate for uplink NOMA is proposed to overcome this problem. The CF-NOMA will obtain a higher chance of justice and interruption than the SIC-NOMA. In specific, it describes how all the Pareto-optimal solutions can be found by the AP with decoding is the same difficulty as SIC decoding. In comparison, separate calculations are performed to show the benefits of CF-NOMA in terms of the average likelihood of break over SIC-NOMA.

Ahmed Al Amin et. al [17] have proposed a multiple access framework of angular velocity movement MIMO to be collective with NOMA that enhances the users as well as integrates the channel capability of the multiple user downlink NOMA. The user and sum potential of the novel NOMA-OAM-MIMO scheme was measured and correlated with existing protocols, namely typical NOMA MIMO and classical orthogonal communication multiplexed OAM-MIMO. NOMA-OAM-MIMO was suggested to improve the channel functionality for NOMA Mobile Interface wireless communication. Outcome based studies 
revealed that revolutionary NOMA-OAM-MIMO technique with amounts of Tx, Rx UCAs, and OAM mode specification (Case 1) has significantly larger device efficiency than standard schemes by using specification with larger numbers of OAM types.

Wali Ullah Khan et. al [18] have introduced a new methodology focused on multi-objective optimization to better spread capital enabling downlink connectivity in multi-user NOMA networks. Besides, the basic optimization approach successfully improves bandwidth and energy utilization when keeping with the QoS standards, sharing the power expenditure, and successively canceling interruptions. Second, devise a standard issue for spectrum and resource improvement and then use dual strategies of depletion to achieve an effective approach. A single-objective NOMA optimization method with high computing power is also presented as a comparison system for the sake of comparison. For potential NOMA systems, spectral and energy efficiency cooperative analysis is valuable. In this context, this study has introduced a unique approach to jointly improve the energy and spectral efficiency of multi-user NOMA systems. Besides, it takes into account a transmission power contact process that has demonstrated that the suggested multiobjective modeling approach works very well for NOMA systems. The matrix factorization system model results are related to the single-objective procedure standard and the conventional OMA technique.

Animesh Kumar Sahu et. al [19] also studied a problem with the unavailability of spectrum resources attributable to accumulated wireless facilities. The author has investigated the problem of bandwidth resources being unavailable due to growing wireless networks. To counter this, a newer intellectual technology called Cognitive Radio has been developed (CR). This technology institutionalizes the use of available spectrum that occur due to under-use of the licensed spectrum. The discovery of the spectrum gaps can be accomplished by the use of a process known as spectrum access. There is a range of problems made by primary CRs in accuracy spectrum access, such as data sparsity issue, tracking, and multi-hop fading. To facilitate the removal of these issues, a current cooperative spectrum sensing model, called CSS, was developed. In this, a particular volume was allocated to each CR involved in the process of categorizing according to consistency. For the initial energy detection system, an adaptive threshold equation was built instead of using the conventional threshold equation that was established using the adaptive threshold equation.

Mohsin Ali et. al [20] have recommended an empirical architecture for finding out the probability of spectrum hole utilization (PSHU) of a CRN with soft cooperative spectrum sensing using a realtime approach of a fixed development framework of CSS. In realistic networks, the period of a time frame is typically defined, where the time period consists of detecting, tracking, and propagation intervals. Growing the period of sensing and monitoring in cooperative spectrum sensing, therefore, increases the likelihood of successful identification of the primary customer (PU) existence or omission, but it reduces number of propagation, resulting in lower PSHU. A sweeping monitoring cycle is necessary as many secondary users (SUs) transmit the information sensed into the fusion center (FC) and/or multiple bits are used for each SU in soft cooperative spectrum sensing. Thus, while considering the overall amount of SUs and tracking bits, processing time often has a related influence on the PSHU.

On Analyzing the various solutions proposed for the problem we understand that holds the following features.

The one-bit measurement in a cognitive radio network was utilized for spectrum sensing. The Likelihood Ratio Testing for one-bit spectrum sensing issues was achieved. The one-bit spectrum sensing is achieved 
as a discrete Gaussian process with autocorrelation procedure is used within instantaneous sequential samples of received information in the cognitive radio network. For one sensor that is then extended to several sensors, a one-bit range sensing criterion is derived. The detector output is evaluated for the likelihood of false alarm and the detection accuracy by acquiring closed-form equations. The proposed onebit LRT predictor with lower complexity has equal accuracy to that of non-one-bit sensors (i.e., quadratic and energy detection systems). The empirical concepts are corroborated by simulated data and affirm the efficiency of the proposed detector in the sense of highly correlated signals.

Even though the system holds the above-specified features we can find that the following are missing.

There is no complete mechanization and for any adjustments to be enforced it requires processing time.

A multi-band transmitter is always required.

Security issue: In spectrum sensing, there are more possibilities available for attackers compared with traditional wireless networks. Without warning, the data can be eavesdropped on or changed. The network could be jammed or overplayed by opponents.

$\square$ Due to its adverse effects, the Quality of Service in Communication systems is impaired.

$\square$ A major challenge in cognitive radio is the interpretation of analyses into actions.

$\square$ Due to problems such as wireless channel inability, receiver ambiguity for decision-making purposes, and so on, system assessment becomes obsolete quickly.

$\square$ Needs continuous data from the participant's user in activities that require a matched filtering function and are considered to become more difficult to obtain, particularly in tactical conditions.

$\square$ Channel interference is high, as the nature of the wireless channel is highly sensitive, particularly for resource-generated information.

\section{Objectives of the Proposed scheme}

To fulfill the missing features this work is carried out with the following objectives. The NOMA powered system is employed for Cognitive Radio Network (CRN) for improving the performance of the network. The cooperative spectrum sensing could be effectively determined in the proposed CRN. The efficient and accurate cooperative spectrum sensing (CSS) is used to progress the capable operation of the cooperative spectrum. The spectrum of primary users can be utilized by the secondary users with the proper acknowledgment of the primary user network. The cooperative spectrum sensing is dependent on the model of sensing of CRN, the sensing time and the latency should be minimized for increasing cooperative users. The efficient and accurate CSS performance should be improved by eliminating interference to the primary and secondary user network, the periodic CSS mode can be effectively achieved by applying MIMONOMA technology. The proposed NOMA is fascinated with significant benefits in CRN is an essential wireless communication method for upcoming $5 \mathrm{G}$ technology.

\section{NOMA CRN model for $5 \mathrm{G}$ wireless communication}

The Non-Orthogonal Multiple Access (NOMA) wireless 5 G networking technology is adapted to the cognitive radio network in the suggested technique (CRN). Cooperative spectrum sensing (CSS) is essential in Cognitive Radio Networks (CRNs) to ensure that opportunistic secondary user (SU) spectrum access does not disrupt established primary users (PUs). For CSS in CRN using NOMA, a novel 
methodology is proposed. The spectrum sensing program helps SU to perform synchronized cooperative spectrum sensing and transmitting, enhancing both spectrum sensitivity and CRN throughput. Cooperative spectrum sensing (CSS) is seen as an effective technique to optimize the use of limited spectrum. In cognitive radio networks, accurate identification of white spaces is essential. Initial studies indicate that precise detection is difficult in numerous primary user and secondary user systems, particularly under severe multipath circumstances. The impact of the AWGN channel and Rayleigh multipath fading channels on cooperative spectrum sensing in a multiple primary user setting is proposed in this research for a preproposed sensor called the spherical detection system using the solution to the eigenvalue. In the absence of channel noise, a greater cooperative spectrum efficiency can be observed. CSS is used to maximize the utilization of effective spectrum. Registered main user spectrum bands are shared with secondary users through the synchronized transmission of information to primary users. Under the conditions, the cooperative spectrum sensing ensures that the separate channel interference can be expected to be marginal to the primary user than the agreed threshold meaning. Using wireless channels such as AWGN and Rayleigh fading channels, the MIMO NOMA is used in CRN. The proposed NOMA is delighted by the considerable advantages of $\mathrm{CRN}$, an important form of wireless networking for upcoming $5 \mathrm{G}$ wireless technologies.

NOMA CRN Model for 5G wireless communication tries to fulfill the following

It provides greater use and efficacy of the spectrum.

It strengthens connection efficiency.

It is smaller in cost.

It uses specialized typologies for networks.

It has a basic network configuration.

It is simple to customize and easy to download.

There's less complexity to it.

A core feature of cognitive radio is mutual spectrum sensing to avoid harmful interaction with licensed users and to define the licensed bandwidth to improve the use of the bandwidth.

$\square$ Cognitive radios can conduct cooperative spectrum sensing, classify unused spectrum creatively, and use certain spectrum gaps disingenuously for their communication.

$\square$ The main idea proposed to be part of the fifth-generation cellular wireless standards is cognitive radio technology $(5 \mathrm{G})$.

For the successful implementation of CR networks, effective energy-sensing is essential.

Through leveraging multi-user spatial diversity, collaborative spectrum sensing (CSS) schemes will dramatically increase the sensing performance of CR networks.

$\square$ Due to the usage of multiple users on the same frequency services, it provides greater spectral performance.

$\square \quad$ It provides huge networking by supporting more needs at the same time simultaneously.

$\square$ Due to continuous delivery, it provides lower latency all the time instead of a dedicated planned time slot.

$\square \quad$ It provides all users with improved Quality of Service using scalable algorithms for power management. It aims to maximize cell-edge throughput and to enhance the cell-edge user experience.

The enhanced output is given by NOMA along with MIMO. 
The key purpose is to assess device performance in terms of how the NOMA proposed algorithm maintains secondary target user performance. As an incomplete CSI, the second obstacle in this planned CR network needs to be explored because the relay is unable to gain wireless channel awareness. This study's significant advantages are summarized as follows:

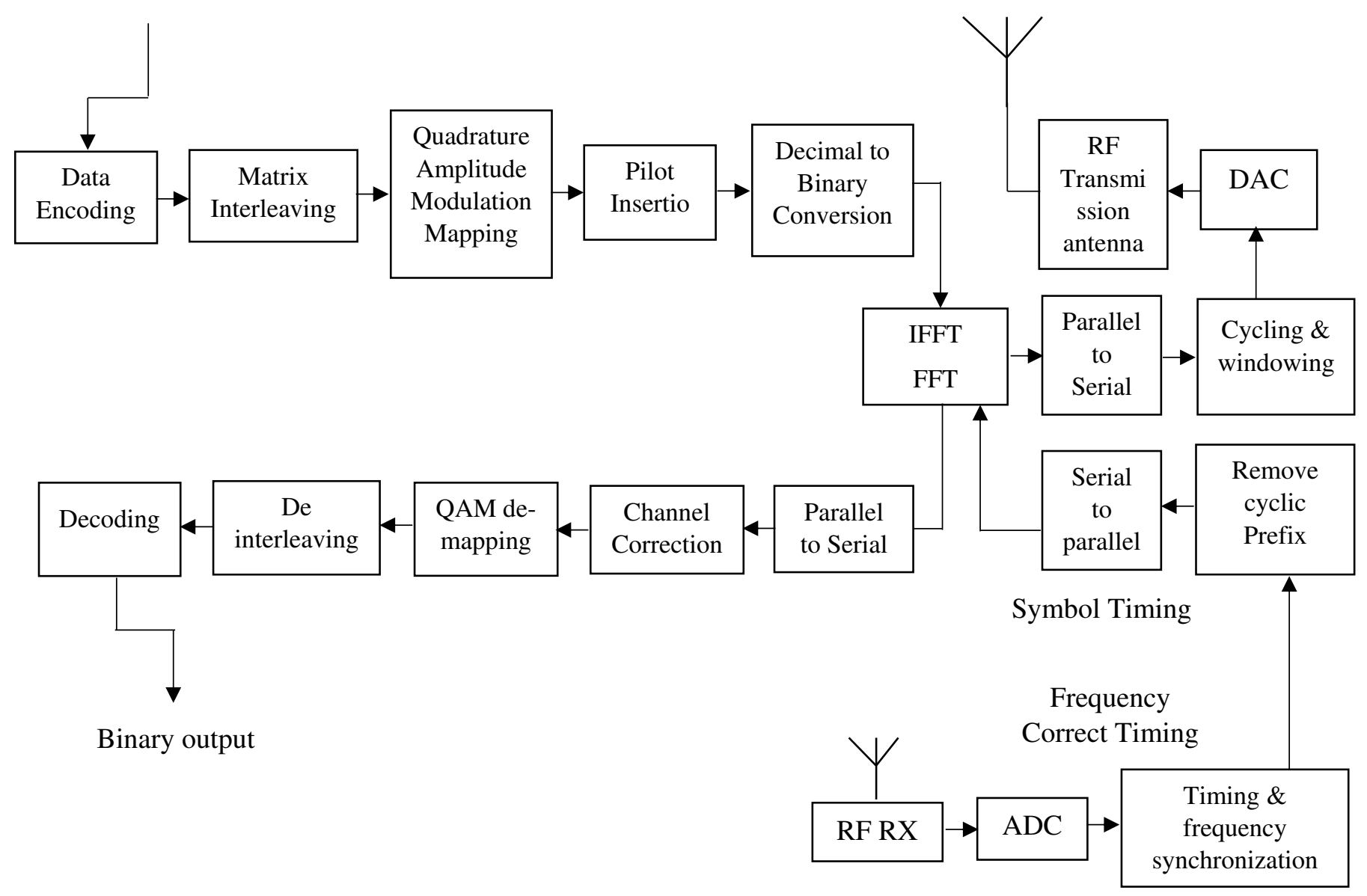

Figure 1: Architecture diagram of NOMO CRN for imperfect Channel State Information (CSI)

1. A CR scheme is suggested that includes a backup network composed of secondary subscribers (SU) to use the $5 \mathrm{G}$ spectrum in two circumstances, namely the availability and non-availability of the primary user (PU). SU can use the NOMA system to connect with two destinations and this framework can dramatically increase the usage of the spectrum relative to the usual NOMA.

2. The effect of incomplete CSI on device assessment was not well studied. First, by identifying the near user as a relay, a strategy is suggested to achieve enhanced efficiency of the far user. Such a benefit of a NOMA scheme can be achieved by comparing two versions by running simulations. Also, to achieve objective assessments on the CR network in submission ranges. To the full knowledge of the authors, this is the first study of CR downlink communication that recognizes NOMA collection and incomplete CSI. 
3. The closed-form representations of the likelihood of outage are obtained from us. To have a perspective on such a CR network, it is important to analyze each user's exponential outage output under the increasing magnitude of calculation. Besides, designing an approach to achieving optimum NOMA mode outage efficiency estimates, taking into account the differing values of resource allocation variables allocated to access point.

4. Asymptotic terms are implemented to validate this outage occurrence straightforwardly because of the complexity of demonstrating outage output for two users via these closed-form expressions. The approximate outage efficiency is required to meet comparable empirical outcomes at a certain reasonable range.

\section{Mathematical Model of NOMA CRN model for 5G wireless communication}

Let us presume that the existence of $\mathrm{P}$ primary users, where $\mathrm{N}$ observations are obtained, is cohesively sensed by $\mathrm{K}$ secondary users. Thus, alternative hypothesis, $\mathrm{H} 0$ and $\mathrm{H} 1$, are described in the signal algorithm for the existence (null) and the existence (alternative) of the primary user signal, accordingly, as wants to follow:

$$
\begin{aligned}
& \text { Css : y }[n]=\text { ws }[n] \\
& \text { Css } 1: y[n]=h \text { s }[n]+w[n]:
\end{aligned}
$$

Here, $\mathrm{y} ; \mathrm{w} ; \mathrm{h}$ and s represent simultaneously the distinct samples obtained, noise, a factor of scaling function, and main signal conveyed. No signal is observed below H0, as in (1), and the samples obtained are nothing more than the Gaussian noise additive complex; the absence of the primary user is then announced. When under H1, as in (2), in addition to the noise, the samples obtained are signal measurements; thus, the primary user's presence is declared. Subsequently, the analysis variable received is:

$$
\text { Outcr }=\mathrm{Hs}+\mathrm{ws}
$$

Here, $y \in \mathrm{Ck}^{\wedge} \mathrm{K}(\mathrm{x}) \mathrm{Pk} \mathrm{x} \mathrm{N}$ (Input) may be a dimensional complex vector that characterizes the 'P' primary users with the obtaining linear function to sense P. The Kx1vector ' $w$ ' could be a multifaceted White Gaussian noise matrix vector with $N \sim\left(0, \sigma_{w}^{2}\right)$ i.i.d. spread having mean of zero and complicated K sensors

signal - to - noise, however. The network gain parameters are expressed by the ${ }^{\sigma_{w}^{2}}$, element matrix $\mathrm{H}=[\mathrm{h} 1$; $\mathrm{h} 2 ;::: ; \mathrm{hp}]$. For eg, h1 reflects the vector of all streams between the first secondary network and all user equipment (K sensors); likewise, as seen in Figure 1, for all hP entries. As followed, it can be written the $\mathrm{H}$ sequence:

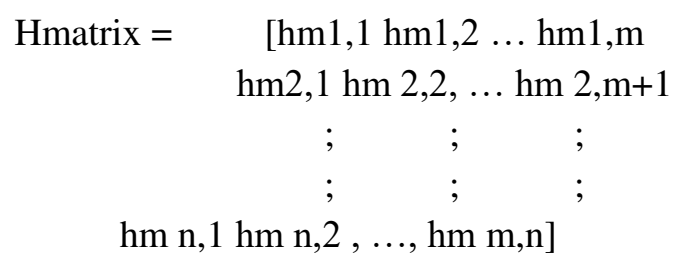

The $\mathrm{P}$ primary user signals are denoted as a $\mathrm{P} \times 1$ matrix vector $\mathrm{A}=[\mathrm{a} 1 ; \mathrm{a} 2 ;:::$; $\mathrm{aP}] \mathrm{T}$, where the samples of primary users signals can be identified with complex Gaussian distribution with zero mean and $\sigma 2 \mathrm{~s}$ 
variance, $\mathbf{s} \sim N\left(0, \sigma_{s}^{2}\right)$ and uncorrelated with Gaussian white noise. The $\mathrm{H}$ index matrix is assigned a constant value during the spectrum sensing duration; thus, it is defined as deterministic. Based on the (3) and (4), the received factors are denoted by a $\mathrm{K} x \mathrm{~N}$ matrix vector $\mathrm{Y}$ as:

$$
\mathrm{H}=[\mathrm{h} 1 ; \mathrm{h} 2 ;::: ; \mathrm{hN}]:(5)
$$

\section{Implementation of Cognitive Radio Network using Non-Orthogonal Multiple Access (NOMA) in imperfect Channel State Information (CSI)}

With respect to the condition of each user's debugging process, the NOMA model is studied. Based on the transmitting signal-to-noise ratio (SNR) at the primary source and interference constraint from the primary network, the closed-form interpretations of the outage probability for two users are acquired considering efficiency as the primary parameter. To gain further ideas, an asymptotic calculation of the outage risk in the high SNR regime is given. Optimum efficiency provides enormous clarity when calculating the power allocation component. As a candidate strategy for potential 5G networks, the NOMA network has recently acquired important coverage. With regard to the state of each user's decoding process, the NOMA model is studied. Based on propagation signal-to-noise ratio (SNR) at the primary source and the constraint of distortion from the primary network, closed-form interpretations of the outage probability for two users are acquired in terms of efficiency. In order to get a clear picture of the system, an asymptotic estimation of the outage risk in the high SNR context is given. Optimum efficiency provides more clarity when calculating the power allocation component. Previously, as a candidate method for future 5G networks, the NOMA network has received substantial attention. To achieve low complexity, the SIC decoding technique aims to address the reliability of the NOMA system in practical NOMA systems, and it requires grouping of users as a major challenge. Contrasting the well-known improved water-filling process, NOMA seeks to provide greater control to users who meet lower channel criteria, and thus the client's justice is fulfilled. Considering recent studies on the NOMA process, the improved usefulness can be attained by using a relaying network with NOMA to incorporate cooperative NOMA.

At the communication transmitter, the cognitive radio network base stations (BS) adopted with $N_{t}$ electromagnetic antennas utilized to communicate the signal to a regular of sole antenna primary users $\mathrm{U}=$ $[1,2,3,4,5, \ldots, \mathrm{U}]$, where $\mathrm{U}<=\mathrm{Nt}, \mathrm{N}$ is the total number of users. The wireless message is obtained by $m^{\text {th }}$ user could be explained as;

$$
y_{m}=h_{m}^{H} \mathbf{x}+\eta_{m}
$$

Where, $h_{m}$ is the CSI channel matrix through BS to $m^{\text {th }}$ CRN user, $\mathrm{x}$ is the communication signal, $\mathbf{x}=\left[\begin{array}{llll}\mathbf{X}_{1} & \mathbf{X}_{2} & \ldots \ldots . . \mathbf{x}_{t}\end{array}\right]$ and white noise $\eta_{m} N\left(0, \sigma_{n m}^{2}\right)$ is Channel State Information (CSI) AWGN at the end of the receiver. The modifications of all the $\mathrm{CRN}$ users could also give to the same, i.e. $\sigma_{n, m}^{2}=\sigma_{n}^{2}$.

Hence wireless signal transmit SNR could be modified as; $\mathrm{snr}=\mathrm{P}_{t} / \sigma_{n}^{2}$ and $\sigma_{n}^{2}$ can be derived for a complete range of SNR, so the SNR in $\mathrm{dB} \rightarrow$ infinity, is equivalent to $\mathrm{P}_{t} \rightarrow \infty$.

In the proposed system model, the BS transmits the interleaved messages with a rate splitting approach. 
The data bits for ' $\mathrm{m}^{\text {th }}$, user can be represented as, $\mathrm{w}_{m, \ell}=\left[\begin{array}{l}\mathrm{b}_{m, 1} \\ \mathrm{~b}_{m, 2}\end{array}\right.$ Each communication can be separated into two parts within the Radio Frequency communication system, namely the private part and the common part. To build a consistent message, the common component of each user's message should be constructed. For two users it can be written as;

$$
\mathrm{W}_{12}=\left\{\mathrm{w}_{1}^{12} \mathrm{w}_{2}^{12}\right\}
$$

Further, the remaining and common part of the messages can be encoded into the autonomous streaming of information; Dt $=[\mathrm{D} 0, \mathrm{D} 1, \mathrm{D} 2, \mathrm{D} 3, \ldots$ Dend $]$. Where Do is the encoded common part of information and Dend is the encoded private parts of the symbol of $\mathrm{m}$ secondary users. These 'end +1 ' information symbols may be clustered in the matrix vector form $\mathrm{Dt}=[\mathrm{D} 0, \mathrm{D} 1, \mathrm{D} 2, \mathrm{D} 3, \ldots \mathrm{Dend}]^{\wedge} \mathrm{T}$ such that,

$$
\mathrm{E}\left\{\mathrm{SS}^{\wedge} \mathrm{H}\right\}=\mathrm{In} .
$$

The symbols are mapped appropriately through the use of a linear precoder, defined as; , before transferring from the base station,

$\mathbf{P}=\left[\mathrm{p}_{\mathrm{c}}, \mathrm{p}_{1}, \ldots \ldots \ldots . \mathrm{p}_{\mathrm{m}}\right]$. Thus, the communication information may be formulated as,

$$
\mathbf{x}=\mathbf{P S}=\mathrm{p}_{\mathrm{c}} \mathrm{S}_{\mathrm{c}}+\sum_{\mathrm{m}=1}^{\mathrm{m}} \mathrm{p}_{\mathrm{m}} \mathrm{S}_{\mathrm{m}}
$$

In addition, the typical communication is transposed over the top of the direct message in RS. The power constraint for this, can be expressed as, $\operatorname{tr}\left(\mathrm{PP}^{\mathrm{H}}\right) \leq \mathrm{P}_{\mathrm{t}}$. The $\mathrm{m}^{\text {th }}$ user received average power can be written as;

$$
\text { pow }_{\mathrm{avg}, \mathrm{m}}=\overbrace{\left|h_{m}^{H} p_{c}\right|^{2}}^{s_{c, \mathrm{~m}}}+\overbrace{\left|h_{m}^{H} p_{m}\right|^{2}}^{s_{m}}+\overbrace{\sum_{i \neq m}\left|h_{m}^{H} p_{i}\right|^{2}+\sigma_{n}^{2}}^{\text {Interference }}
$$

Consider all such communications as interference. Once the specific information is successfully collected, it is filtered using SIC to enhance the private information identification capability. The typical information and private information of AWGN and Raleigh channel signal to interference noise ratio (SINR) can be described as,

$$
\gamma_{m}^{c}=\frac{\mathrm{P}_{\mathrm{c}}\left|\mathrm{h}_{\mathrm{m}}^{\mathrm{H}} \mathrm{W}_{\mathrm{c}}\right|^{2}}{\sum_{\mathrm{j}=1}^{\mathrm{m}} \mathrm{P}_{\mathrm{j}}\left|\mathrm{h}_{\mathrm{m}}^{\mathrm{H}} \mathrm{W}_{\mathrm{j}}\right|^{2}+1}, \gamma_{m}^{p}=\frac{\mathrm{P}_{m}\left|\mathrm{~h}_{\mathrm{m}}^{\mathrm{H}} \mathrm{W}_{m}\right|^{2}}{\sum_{j \neq m} \mathrm{P}_{\mathrm{j}}\left|\mathrm{h}_{\mathrm{m}}^{\mathrm{H}} \mathrm{W}_{\mathrm{j}}\right|^{2}+1}
$$

The realizable amount of common information could be stated as $\mathrm{R}_{\mathrm{c}}^{\mathrm{RS}}=\log _{2}\left(1+\gamma^{c}\right)$, where $\gamma^{\mathrm{c}}=\min \left(\gamma_{\mathrm{m}}^{\mathrm{c}}\right)$ for ensuring that received common information is effectively decrypted by each secondary user. The amount of the private information is stated as $R_{p}^{R S}=\sum_{m=1}^{m} \log _{2}\left(1+\gamma_{m}^{p}\right)$. Then the remaining quantity degree can be expressed as $\mathrm{R}_{\mathrm{sum}}^{\mathrm{RS}}=\mathrm{R}_{\mathrm{c}}^{\mathrm{RS}}+\mathrm{R}_{\mathrm{p}}^{\mathrm{RS}}$.

The channel capacity maximization can be seen as an issue of max-min compatibility enhancement. 
Due to variance in CSI, the real levels are not given as development vectors (channel state information). So the pre-coders are designed for rates attainable under the worst case. The worst case of ' $\mathrm{M}$ ' user's acceptable rates can be represented as,

$$
\mathrm{F}^{\prime}(\mathrm{d}, \mathrm{n})=\operatorname{minima}(\mathrm{F}(\mathrm{d}, \mathrm{n})) \text { with } \mathrm{F}^{\prime}=\operatorname{minima}(\mathrm{Fn}(\mathrm{Hn}))
$$

For the sake of ease, the 'M'th user poor common degree can be explained as D'n

Where,

$$
\Sigma \mathrm{Dn}=\mathrm{Fc}
$$

Thus, whole poorest situation attainable amount could be denoted as

$$
\text { Ftotal,n = Fm' + Dm', }
$$

Thus, to increase the rate of the whole amount of data, the enhancement solution can be written as,

Maxima ( $\mathrm{F}$ total)

With respect to,

F'm + D'm $=$ Ftotal

D'm $>=0$

$\operatorname{Total}\left(\mathrm{PP}^{\wedge} \mathrm{H}\right)<=$ Ptotal

In addition, the signal is identified and projected with the support of SIC at the receiving antenna. In accumulation to quantity separating, the proposed SIC methodology in NOMA similarly gives roughly additional benefits, such as; the convergence time in frequency modulation is quicker in SIC-based NOMA and user organizing could be enhanced based on channel maximization of SINR. Furthermore, it supports the approximate response and feedback to the controller. Consequent operations are done by the decoder (DEC) (for ease, supposed QAM modulation).

(A) Acquired calculated usual signal $\left\{\hat{\mathrm{d}}_{\mathrm{m}}(\mathrm{i})\right\}$ based $\left\{\hat{\varphi}_{\mathrm{m}}(\mathrm{j})\right\}$ (estimated through SIC identification). This is calculated that all computed $\left\{\hat{\varphi}_{\mathrm{m}}(\mathrm{j})\right\}$ are irrelevant (that is roughly correct among interleaving). It is strong that $\varphi_{m}(j)=C_{m}\left(\pi_{m}(j)\right)$, where $\pi_{m}(j)$ the arbitrary is interleaved. So, the easy approximation of $\mathrm{d}_{\mathrm{m}}(1)$ can be calculated through $\left\{\hat{\varphi}_{\mathrm{m}}(\mathrm{j})\right\}$.

$$
\mathrm{L}\left(\mathrm{d}_{\mathrm{m}}(1)\right)=\sum_{j=1}^{S} \hat{\varphi}_{\mathrm{m}}(\mathrm{j})
$$

(B) Complete typical APP decryption based on $\left\{\mathrm{L}\left(\mathrm{d}_{\mathrm{m}}(\mathrm{i})\right)\right\}$ as the input data to create a posteriori LLRs (maximum log likelihood ratios) $\left\{\mathrm{L}_{A P P}\left(\mathrm{~d}_{\mathrm{m}}(\mathrm{i})\right)\right\}$ for $\left\{\mathrm{d}_{\mathrm{m}}(\mathrm{i})\right\}$.

(C) Based on few calculations and turbo data processing the output signal of the decryption could be assumed as; 


$$
\mathrm{e}_{\mathrm{DEC}}\left(\hat{\varphi}_{\mathrm{m}}(\mathrm{j})\right) \approx \mathrm{L}_{A P P}\left(\mathrm{~d}_{\mathrm{m}}(1)\right), \mathrm{j}=1,2 \ldots \ldots . . \mathrm{S}
$$

\section{Experimental Setup}

Experimental setup of cognitive radio network (CRN) using non-orthogonal multiple access (NOMA) is achieved by utilizing wireless communication toolbox incorporation with MATLAB 2018. Analytics tools such as graphs and equations, algorithms to improve experiment results, and the ability to maintain methods to assess results are given for experiment setup. Experiment setup preserves a record of the results of the experiment to increase reliability every time an experiment is performed

\section{ASSUMPTIONS}

In this research, few assumptions were considered.

1. We segment binary serial data signal multiple lower-speed sub-band signal for improving the mobility of data through various modules of experiments.

2. Pilot data sub-carriers are inserter in transmitter to reduce errors in frequency and phase transfer.

3. The cyclic-prefix is applied with windowing technique to avoid Inter Symbol Interference (ISI) and Inter Carrier Interference (ICI).

4. The overlapping of frames are restricted.

The various parameters are required to implement the CRN NOMA for efficient and accurate wireless communication for primary and secondary users are given in Table 1.

Table 1: Parameters applied for CRF MIMO NOMA

\begin{tabular}{|l|c|}
\hline \multicolumn{1}{|c|}{ CRN MIMO NOMA Parameters } & Achieved Values \\
\hline CRN Subcarrier data & 256 \\
\hline Pilot data subcarrier & 32 \\
\hline Spacing of Channel State Information $(\mathrm{CSI})$ & $32 \mathrm{MHz}$ \\
\hline Spacing $\left(\Delta_{\mathrm{f}}\right)$ of Carrier frequency Offser & $512 \mathrm{KHz}$ \\
\hline Cooperative Spectrum Sensing & $32.64 \mathrm{MHz}=(1024 \mathrm{kHz} \times 52)$ \\
\hline Interval of Guard Bit & $0.8 \mu \mathrm{sec}$ \\
\hline Symbol Period & $2.2 \mu \mathrm{sec}\left(=1 / \Delta_{\mathrm{f}}\right)$ \\
\hline
\end{tabular}

The specifications of CRN NOMA for efficient and detailed implementation are outlined in Table 2. For other numbers of arrays, the modulation style may be modified to operate for other techniques of synchronization. 
Table 2 CRN NOMA Parameters achievements

\begin{tabular}{|l|c|}
\hline \multicolumn{1}{|c|}{ List of Parameters } & List of Requirements \\
\hline Choice of CRN module & $\begin{array}{c}16 \text { bits length and } 512 \\
\text { frequency in } \mathrm{MHz}\end{array}$ \\
\hline Total number BS & 8 \\
\hline Total number of nodes & 128 \\
\hline Wireless communication & Standard \\
\hline Antenna polarization & 360 degree bidirectional \\
\hline Type of Modulation & $\begin{array}{c}512 \text { Array - Quadrature } \\
\text { Amplitude Modulation }\end{array}$ \\
\hline
\end{tabular}

The correlation among ergodic capability enhancement for AWGN and Rayleigh fading channels is shown in Table 3. There is inconsistent noise on the AWGN channel. This provides comparatively less power than channel Rayleigh.

Table 3 AWGN and Rayleigh channels capacity comparison

\begin{tabular}{|c|c|c|}
\hline $\begin{array}{c}\text { Channel } \\
\text { maximization } \\
\text { in SNR in } \\
\text { decibel }\end{array}$ & $\begin{array}{c}\text { Ergodic Capacity of } \\
\text { AWGN channel in } \\
\text { Mbps }\end{array}$ & $\begin{array}{c}\text { Ergodic Capacity of } \\
\text { Rayleigh channel in } \\
\text { Mbps }\end{array}$ \\
\hline 0 & 10.8387 & 15.9894 \\
\hline 5 & 12.9387 & 16.3489 \\
\hline 10 & 14.9389 & 17.3982 \\
\hline 15 & 16.3873 & 19.3993 \\
\hline 20 & 18.3788 & 21.3984 \\
\hline 25 & 20.3832 & 24.3989 \\
\hline 30 & 22.3973 & 26.3989 \\
\hline 35 & 24.3893 & 28.3938 \\
\hline 40 & 26.3899 & 30.8479 \\
\hline 45 & 28.3833 & 33.3894 \\
\hline 50 & 30.3789 & 36.8994 \\
\hline
\end{tabular}

\section{Results and discussions}

The performance results are discussed using graphical representation and parameters statistical information in this section. The quantitative results are discussed in this section. Empirical results are validated using graphical and statistical representations. In NOMA terminology, it is calling a user detection process. U1 is expected to alert the nearest source before sending input information to the primary source to choose NOMA mode.

Figure 2 shows MIMO NOMA subcarrier generation for CRN. The performance of CRN in secondary networks as utilizing non-orthogonal multiple access (NOMA) is examined. The transmission quality is considered from the to the far NOMA users who expect multiple access to the core network. Figure 2 shows MIMO NOMA using the Rayleigh Fading channel for CRN. The Bit Error Rate Vs Signal to Noise Ratio 
in $\mathrm{dB}$ is measured for both long distance users and short distance users by estimating simulation and theoretical system.

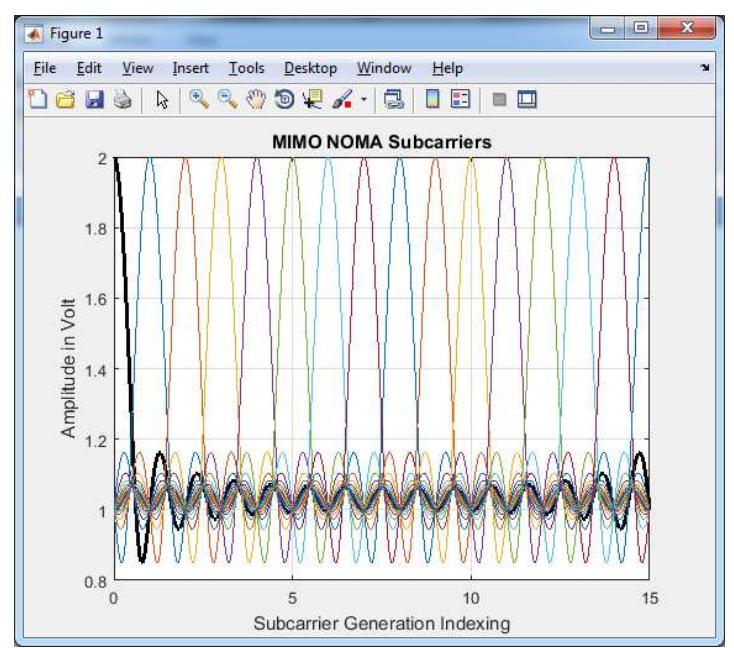

Figure 2: MINO NOMA Subcarrier generation for cognitive radio network

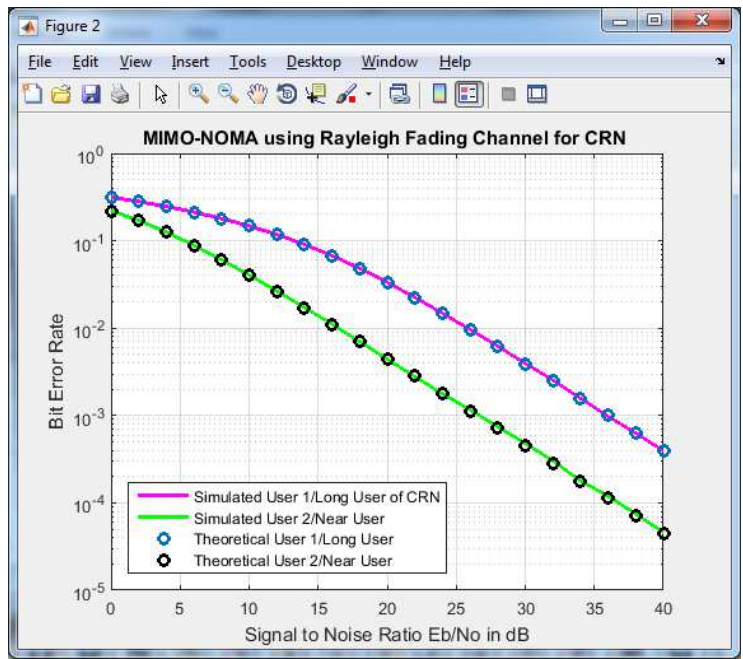

Figure 3: MIMO NOMA using Rayleigh Fading channel for Cognitive Radio Network 


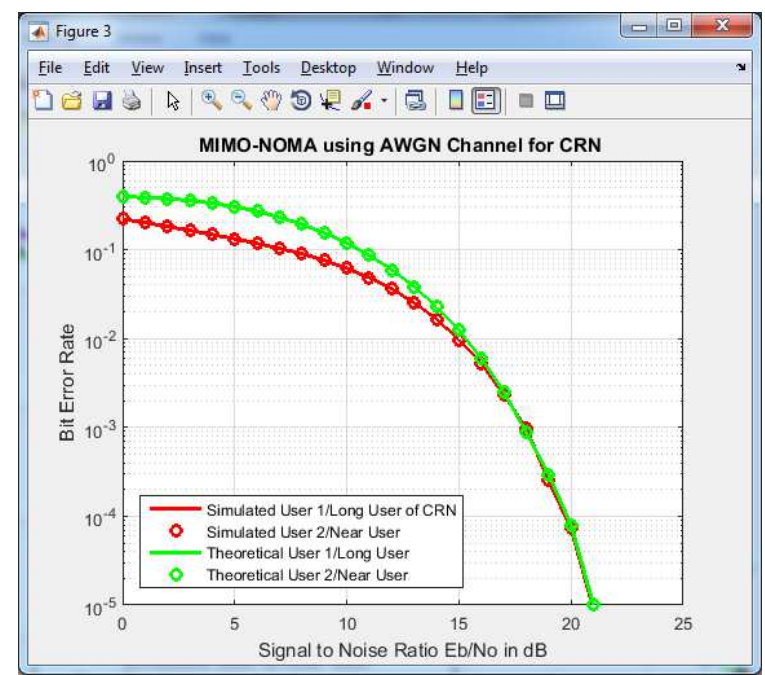

Figure 4: MIMO-NOMA using AWGN Channel for CRN

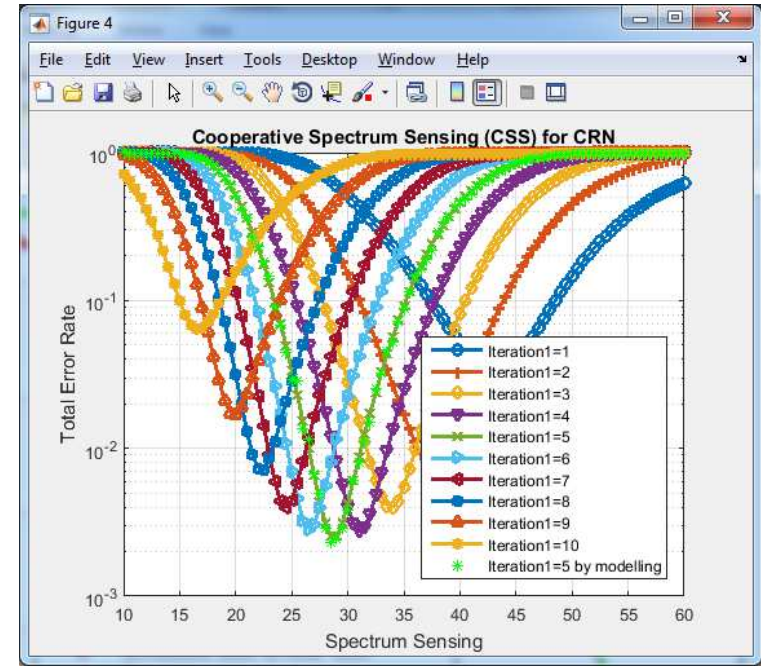

Figure 5: Cooperative Spectrum sensing for CRN using MIMO NOMA

Figure 4 shows the MIMO NOMA using the AWGN channel for CRN as imperfect CSI. Figure 5 shows cooperative spectrum sensing (CSS) using MIMO NOMA to improve the secondary user's spectrum by utilizing the available spectrum of primary users. 


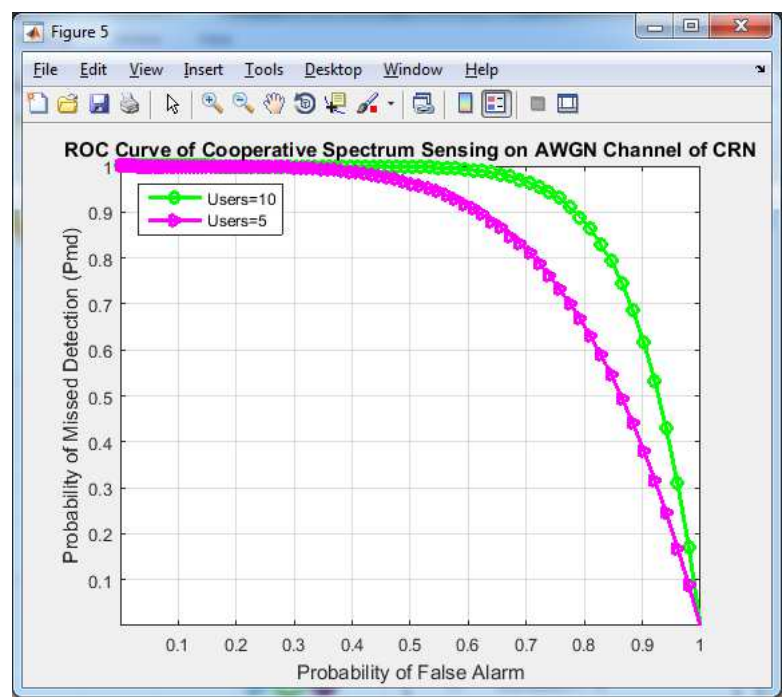

Figure 6: ROC curve of cooperative spectrum sensing for AWGN channel with different number of users

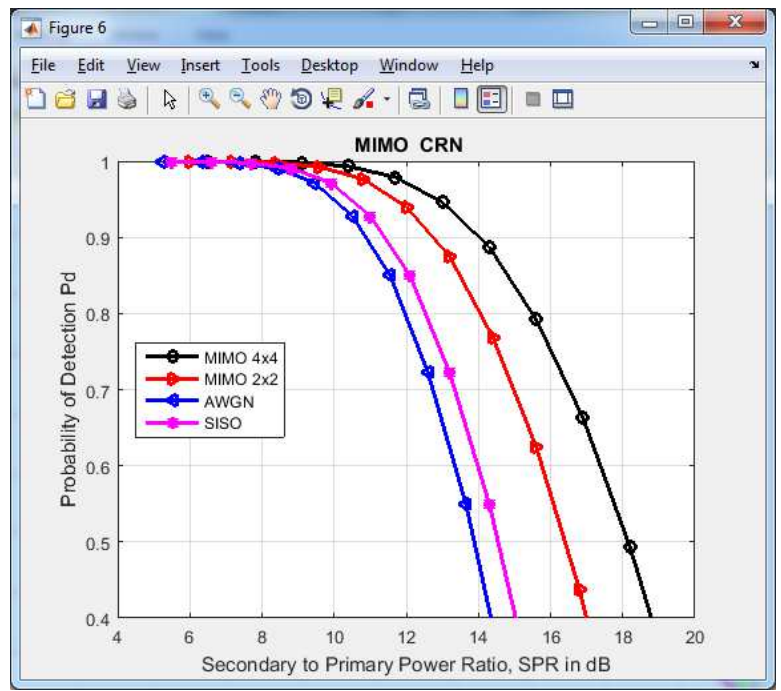

Figure 7: The power ratio estimation for MIMO NOMA CRN

Figure 6 shows the ROC curve of CSS for AWGN channel CRN by improving SNR and capacity in Mbps to improve the number of users. The secondary to primary network power ratio is plotted for different MIMO combinations of NOMA for CRN. Power ration estimation for MIMO NOMA CRN is shown in Figure 7. The proposed analysis contributes by improving the outage performance of far NOMA end-users. The signal to noise ratio (SNR) is improved to discover separated signals for each user, and exact expressions of outage probability are examined in the assumption that the receiver can delete the interference with successive interference elimination completely. 


\section{Conclusion}

Efficient cooperative spectrum sensing (CSS) in Cognitive Radio Network (CRN) using MINO NOMA technology is implemented. The CSS is used to improve the performance of spectrum utilization of primary and secondary users. The available spectrum of primary users can be utilized by the secondary users by proper authentication. The ergodic capacity of CRN among AWGN and Rayleigh Fading channels is improved. This phenomenon is used to improve the spectrum utilization of secondary users. The experimental results prove that CSI imperfections are rectified in NOMA communication to improve the CRN performance.

\section{Declarations:}

1) Funding (information that explains whether and by whom the research was supported)

\section{Not Applicable}

2) Conflicts of interest/Competing interests (include appropriate disclosures)

- All authors have participated in (a) conception and design, or analysis and interpretation of the data; (b) drafting the article or revising it critically for important intellectual content; and (c) approval of the final version.

- This manuscript has not been submitted to, nor is under review at, another journal or other publishing venue.

- The authors have no affiliation with any organization with a direct or indirect financial interest in the subject matter discussed in the manuscript

3) Availability of data and material (data transparency)

\section{Not Applicable}

4) Code availability (software application or custom code)

\section{Not Applicable}

5) Authors' contributions (optional: please review the submission guidelines from the journal whether statements are mandatory)

\section{Not Applicable}




\section{References}

1. Xiao Yang, Min Sheng, Hongguang Sun,Xijun Wang and Jiandong Li, "Spatial Throughput Analysis and Transmission Strategy Design in Energy Harvesting Cognitive Radio Networks", DOI 10.1109/TCOMM.2018.2865001.

2. Giovanni Baggio, Riccardo Bassoli and Fabrizio Granelli, "Cognitive Software-Defined Networking Using Fuzzy Cognitive Maps”, DOI 10.1109/TCCN.2019.2920593

3. Yunfei Chen, Yue Wu, Jie Zhang, Ning Chen, "New Estimators for Primary Channel Gain in Cognitive Radio Networks", IEEE 2018.

4. Gyeongrae Im, Jae Hong Lee, "Outage Probability for Cooperative NOMA Systems With Imperfect SIC in Cognitive Radio Networks", DOI 10.1109/LCOMM.2019.2903040, IEEE 2018.

5. Meng Zheng, Chuqing Wang, Manyi Du, Lin Chen, Wei Liang and Haibin Yu, "A Short Preamble Cognitive MAC Protocol in Cognitive Radio Sensor Networks", IEEE 2018.

6. Zhengqiang Wang, Chenchen Wen, Zifu Fan and Xiaoyu Wan, “A Novel Price-Based Power Allocation Algorithm in Non-Orthogonal Multiple Access Networks", DOI 10.1109/LWC.2017.2768367, IEEE 2017.

7. Ahmed A Amin and Soo Young Shin, "Channel Capacity Analysis of Non-orthogonal Multiple Access with OAM-MIMO System”,DOI 10.1109/LWC.2020.2994355, IEEE 2020.

8. Mina Baghani, Saeedeh Parsaeefard, Mahsa Derakhshani, Walid Saad, "Dynamic NonOrthogonal Multiple Access (NOMA) and Orthogonal Multiple Access (OMA) in 5G Wireless Networks", 2019.

9. Sultangali Arzykulov, Galymzhan Nauryzbayev, Theodoros A. Tsiftsis, "On the Outage of Underlay CR-NOMA Networks with Detect-and-Forward Relaying", DOI 10.1109/TCCN.2019.2916561, IEEE 2019.

10. Dinh-Thuan Do, Anh-Tu Le and Byung Moo Lee, "On Performance Analysis of Underlay Cognitive Radio-Aware Hybrid OMA/NOMA Networks with Imperfect CSI", doi:10.3390/electronics8070819, 2019.

11. Athanasios Papadopoulos, Nestor D. Chatzidiamantis, "Network Coding Techniques for Primary-Secondary User Cooperation in Cognitive Radio Networks", IEEE 2020.

12. Chaoying Yuan, Xiaofeng Tao , $\mathrm{Na} \mathrm{Li} \mathrm{,} \mathrm{"Analysis} \mathrm{on} \mathrm{Secrecy} \mathrm{Capacity} \mathrm{of} \mathrm{Cooperative} \mathrm{Non-}$ Orthogonal Multiple Access With Proactive Jamming”, IEEE 2019.

13. Muhammad Ahsen, Syed Ali Hassan, "Propagation Modeling in Large-Scale Cooperative Multi-Hop Ad Hoc Networks", IEEE 2016.

14. Muhammad Farhan Sohail, Chee Yen Leow, "Energy Efficient Non-Orthogonal Multiple Access for UAV Communication System", DOI 10.1109/TVT.2019.2939186.

15. Zhiyuan $\mathrm{Yu}$, Chao Zhai, "Cooperative Relaying Based Non-Orthogonal Multiple Access (NOMA) With Relay Selection", IEEE 2018.

16. Cheng Guo, Abdelmalik N. Aljalaiy, Chen Fengz, Liqiang Zhao, Victor C. M. Leungy and Rabab K. Ward, "Compute-and-Forward for Uplink Non-Orthogonal Multiple Access", DOI 10.1109/LWC.2018.2844852.

17. Ahmed A Amin and Soo Young Shin, "BChannel Capacity Analysis of Non-orthogonal Multiple Access with OAM-MIMO System”, DOI 10.1109/LWC.2020.2994355, IEEE 2020.

18. Wali Ullah Khan, Furqan Jameel, Tapani Ristaniemi, Shafiullah Khan, Guftaar Ahmad Sardar Sidhu and Ju Liu, "Joint Spectral and Energy Efficiency Optimizationfor Downlink NOMA Networks", DOI 10.1109/TCCN.2019.2945802, IEEE 2019.

19. Animesh Kumar Sahu, Anupama Singh, Nandakumar S, "Improved Adaptive Cooperative Spectrum Sensing in Cognitive Radio Networks", IEEE 2018.

20. Mohsin Ali and Haewoon Nam, "Optimization of Spectrum Utilization in Cooperative Spectrum Sensing”, Sensors 2019, 19, 1922; doi: 10.3390/s19081922. 


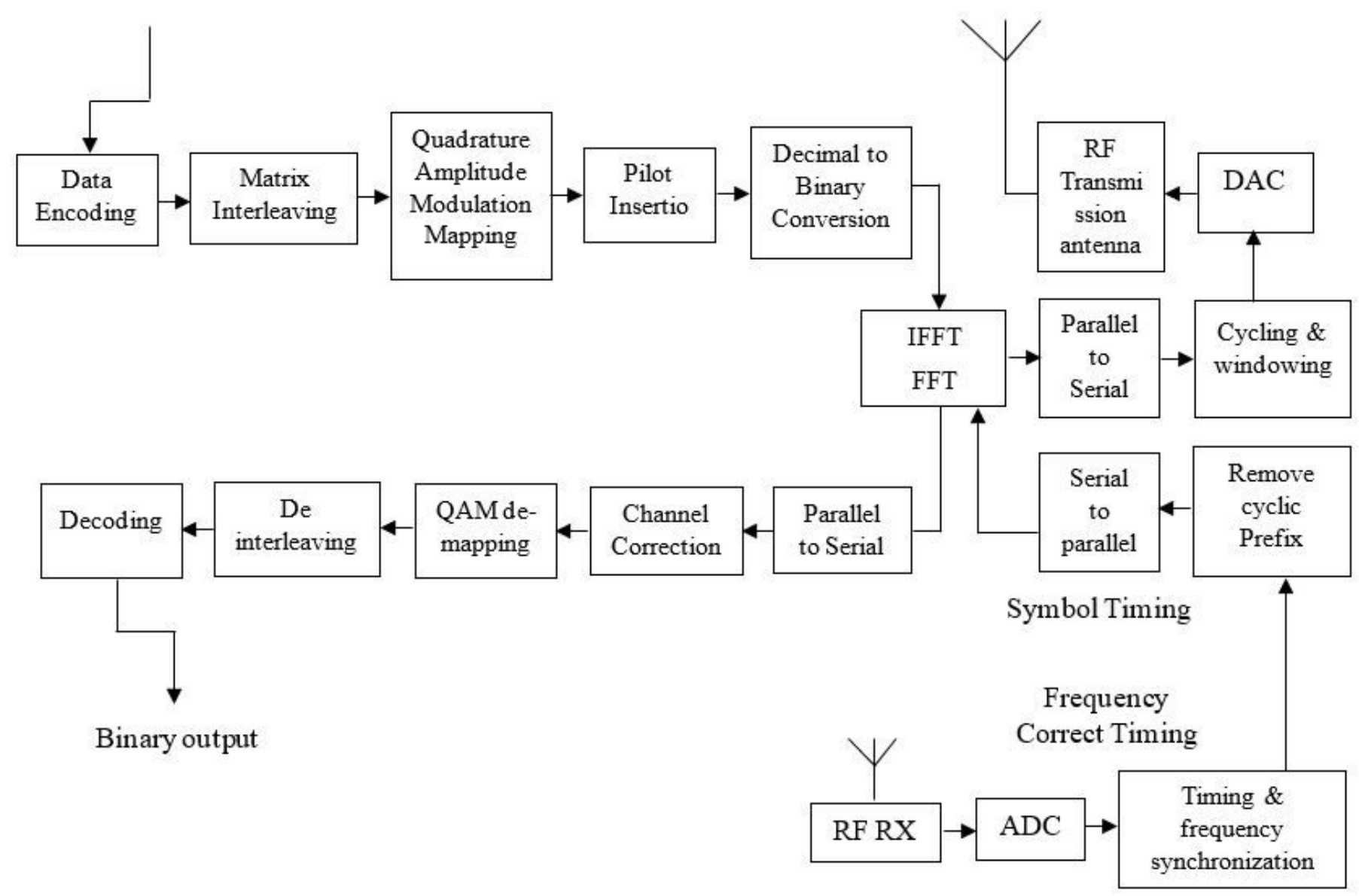

Figure 1

Architecture diagram of NOMO CRN for imperfect Channel State Information (CSI) 


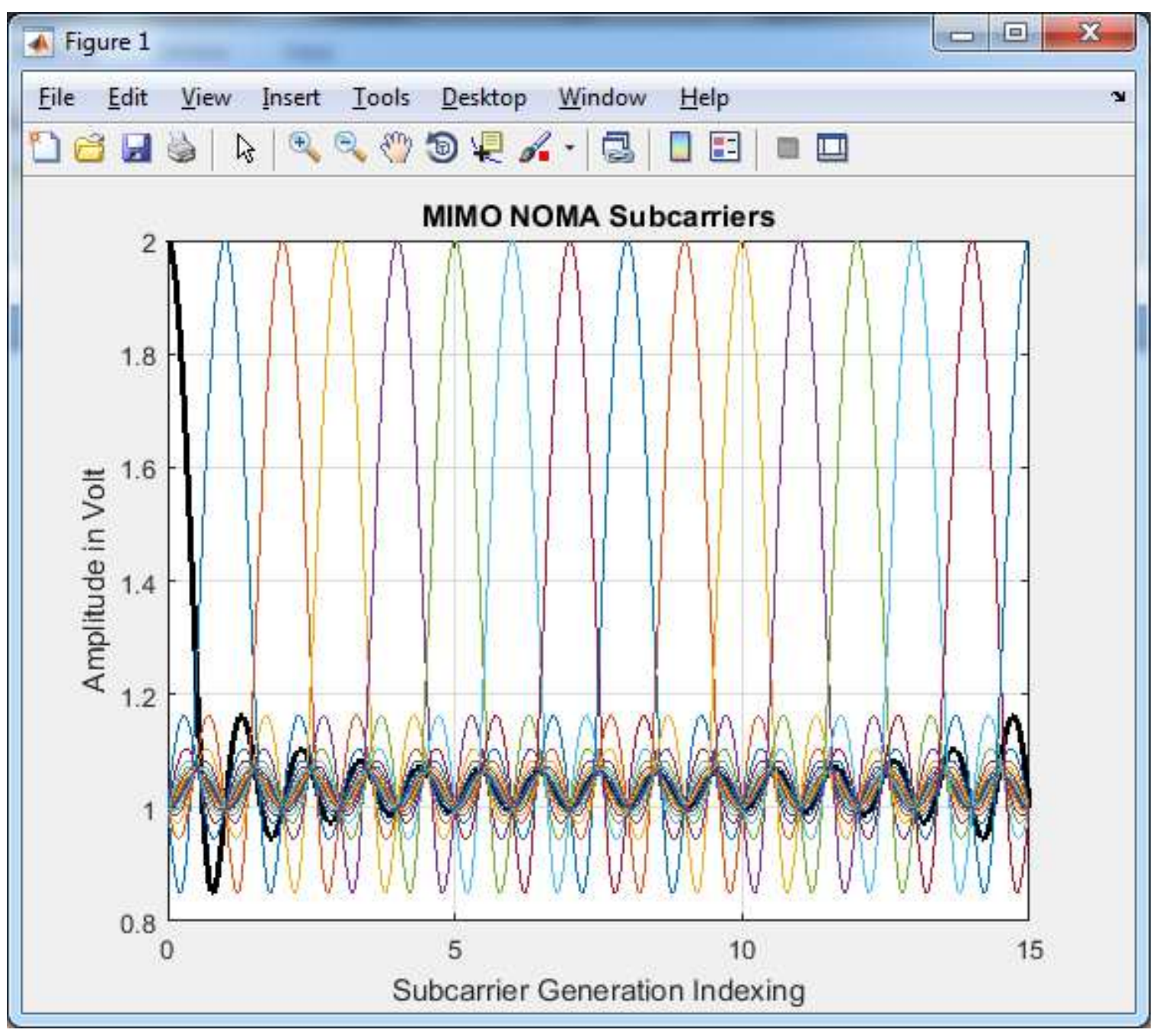

Figure 2

MINO NOMA Subcarrier generation for cognitive radio network 


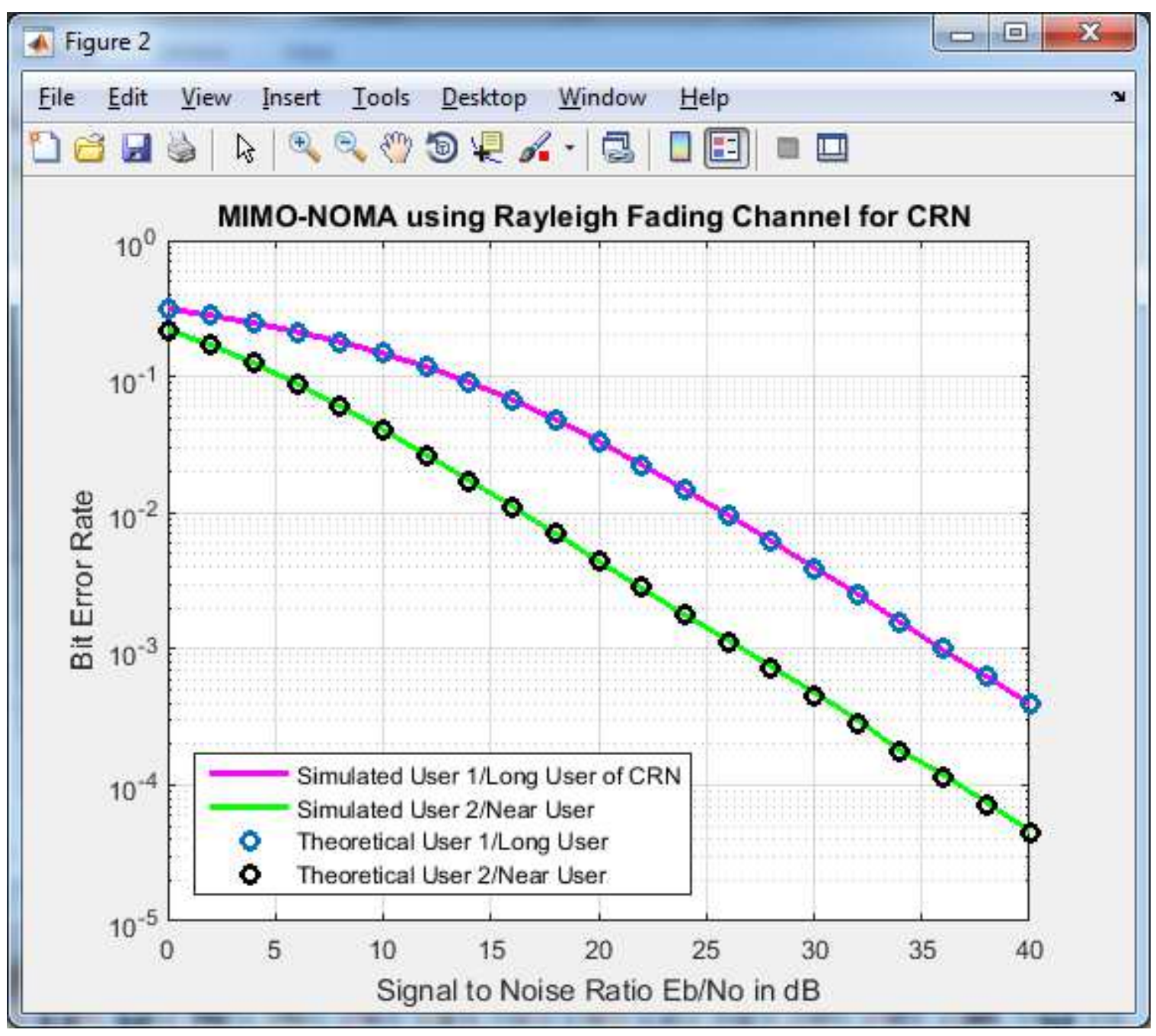

Figure 3

MIMO NOMA using Rayleigh Fading channel for Cognitive Radio Network 


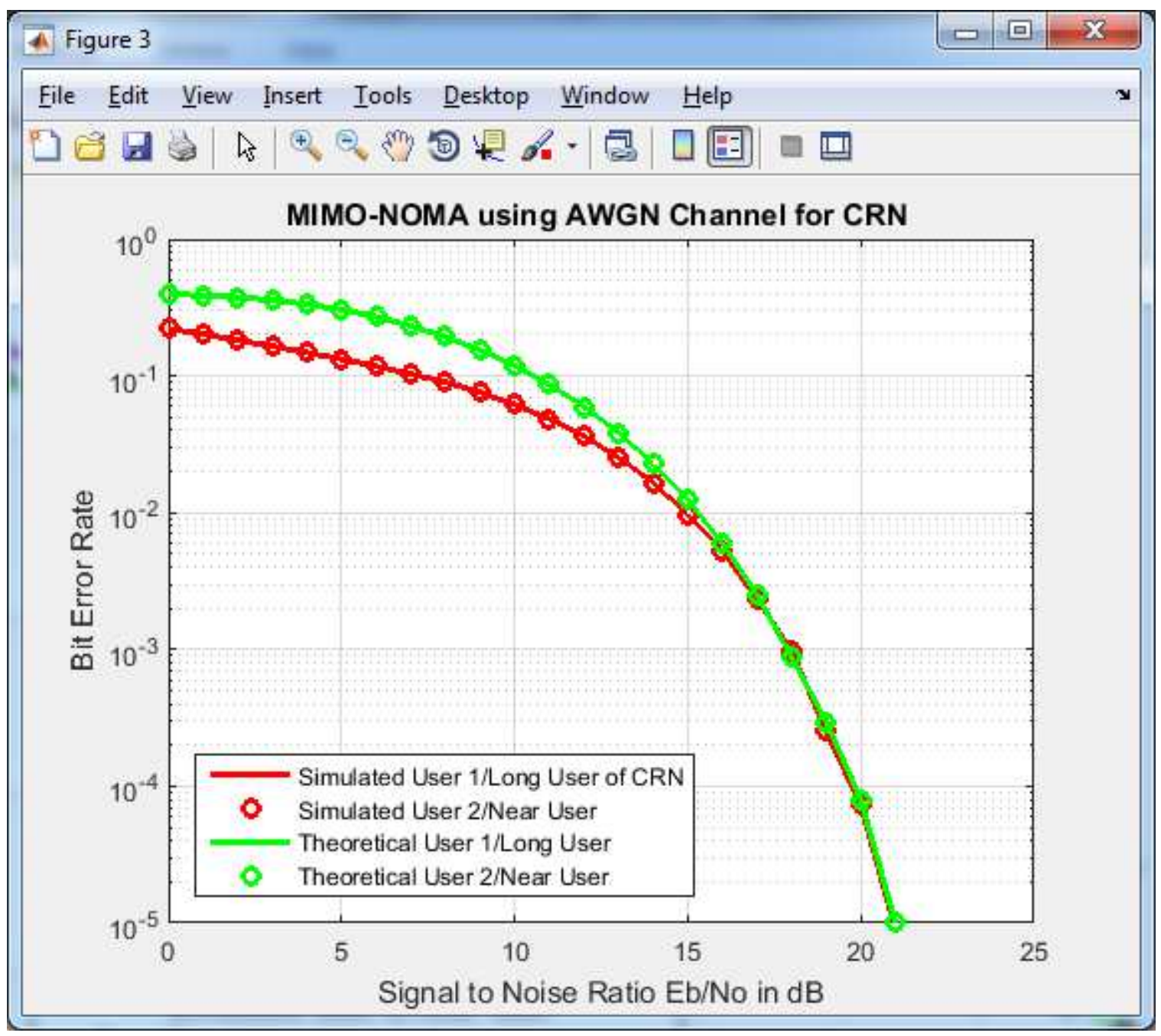

Figure 4

MIMO-NOMA using AWGN Channel for CRN 


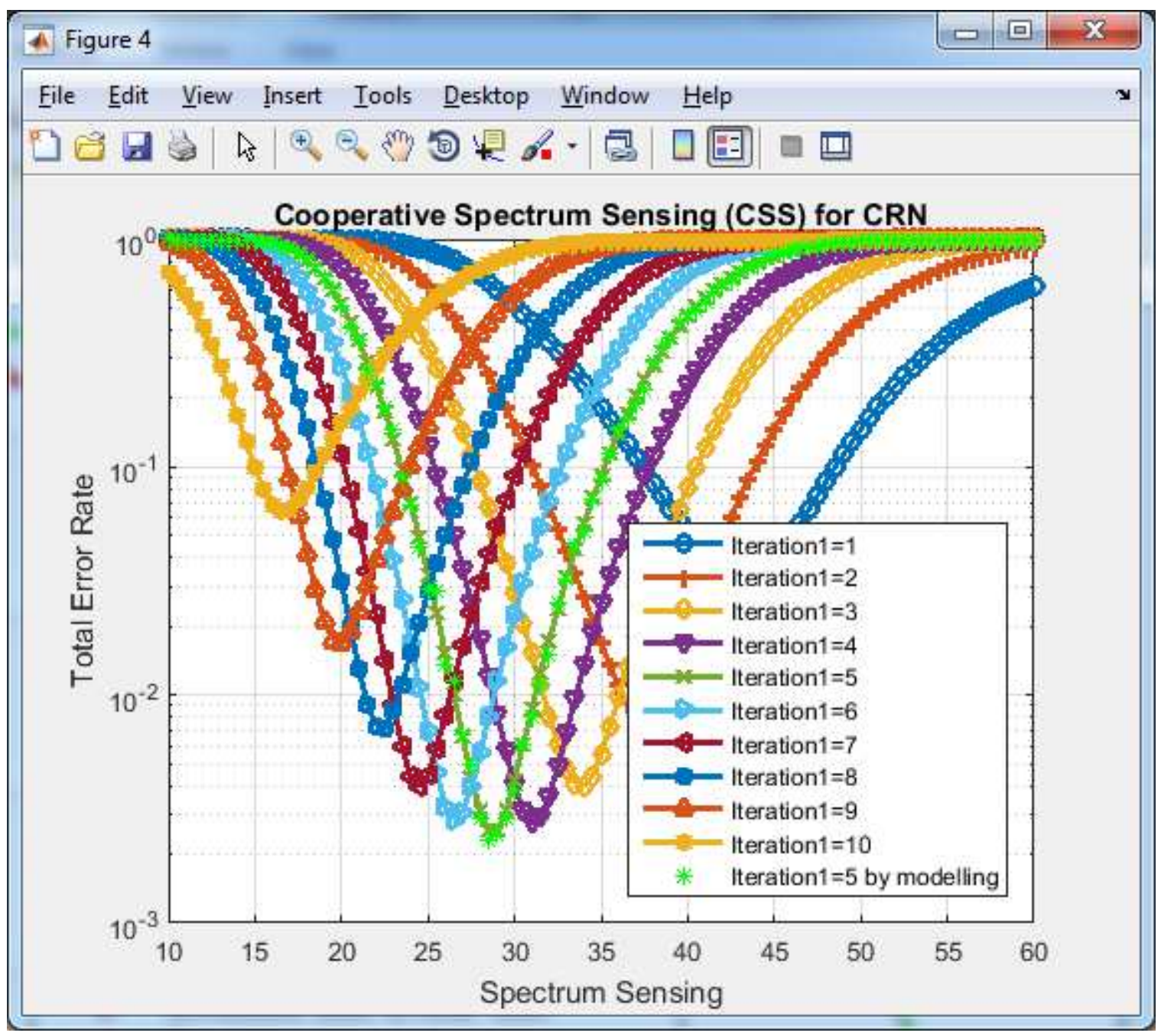

Figure 5

Cooperative Spectrum sensing for CRN using MIMO NOMA Figure 4 shows the MIMO NOMA using the AWGN channel for CRN as imperfect CSI. Figure 5 shows cooperative spectrum sensing (CSS) using MIMO NOMA to improve the secondary user's spectrum by utilizing the available spectrum of primary users. 


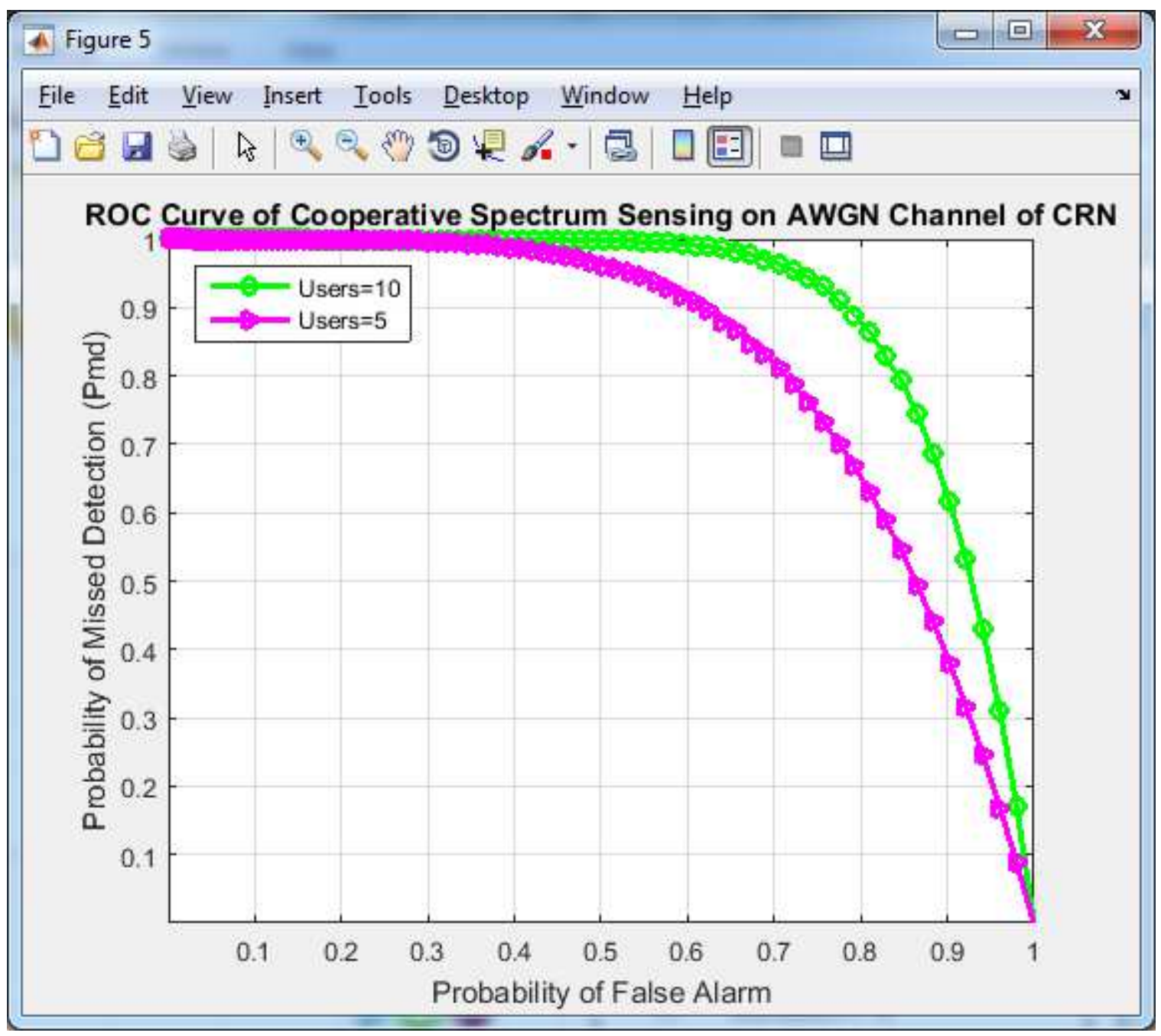

Figure 6

ROC curve of cooperative spectrum sensing for AWGN channel with different number of users 


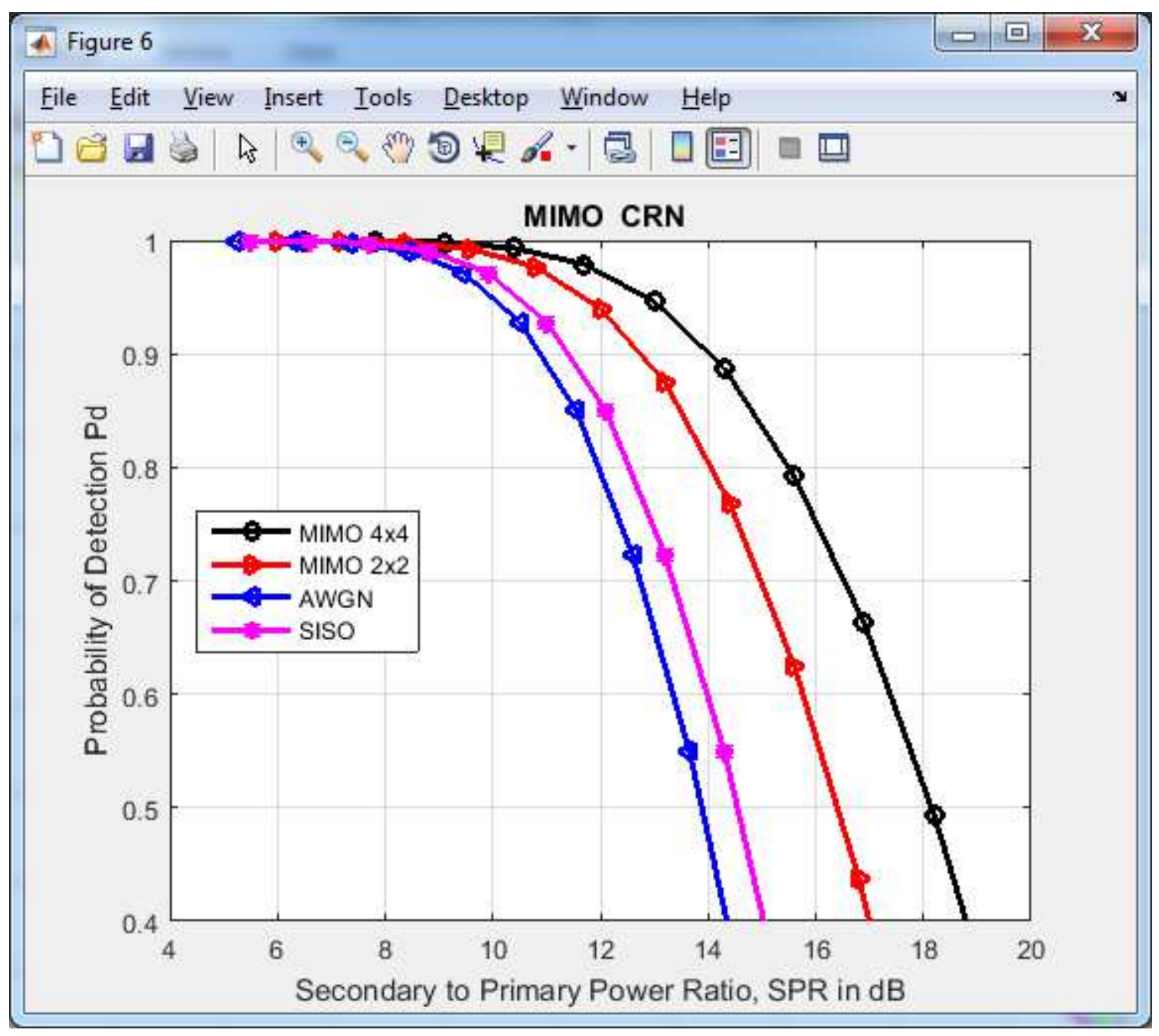

\section{Figure 7}

The power ratio estimation for MIMO NOMA CRN Figure 6 shows the ROC curve of CSS for AWGN channel CRN by improving SNR and capacity in Mbps to improve the number of users. The secondary to primary network power ratio is plotted for different MIMO combinations of NOMA for CRN. Power ration estimation for MIMO NOMA CRN is shown in Figure 7. The proposed analysis contributes by improving the outage performance of far NOMA end-users. The signal to noise ratio (SNR) is improved to discover separated signals for each user, and exact expressions of outage probability are examined in the assumption that the receiver can delete the interference with successive interference elimination completely. 